Results The mean \pm SD was desire $162.9 \pm 10.02$ arousal 143.4 \pm 8.6 lubrification $213.7 \pm 13.4$ orgasm $214.4 \pm 14.8$ satisfaction $222.4 \pm 19.9$ pain $165.6 \pm 11.7$

Conclusions The FSFi in SLE patients are decreased when compared with what is reported in placebo populations but its magnitude is reduced when compared with results reported in other severe chronic diseases. This findings suggests that further studies are needed to find out if belimumab treated SLE patients have some degree of protective effect in sesxual dysfunction.

Funding Source(s): Funding source Institutional and GSK.

\section{COGNITIVE DYSFUNCTION IN AI-CTD IS ASSOCIATED WITH TRADITIONAL CARDIOVASCULAR RISK FACTORS BUT NOT IMMUNOLOGICAL PARAMETERS}

${ }^{1}$ Marta Aguilar Zamora*, ${ }^{2}$ Katie Dutton, ${ }^{2}$ Zoe Wigston, ${ }^{2}$ Adewonuola Alase, ${ }^{2}$ Antonios Psarras, ${ }^{2}$ Md Yuzaiful Md Yusof, ${ }^{2}$ Edward Vital. ${ }^{1}$ Hospital Universitario Dr. Peset, Valencia. Fundación Valenciana de Reumatologia; ${ }^{2}$ University of Leeds

\subsection{6/lupus-2019-Ism.280}

Background Cognitive dysfunction is a common problem in autoimmune connective tissue diseases such as SLE that can be screened for using the Montreal Cognitive Assessment (MoCA). The causes of cognitive dysfunction are poorly understood. They may include immune-mediated neurological dysfunction (which should be targeted using immunosuppression) or traditional cardiovascular risk factors (which may be treated as in non-SLE patients). The purpose of this study was to explore these counter-hypotheses in patients with established autoimmune disease by analysing conventional measures of autoimmunity, cardiovascular risk, as well as validated scores for interferon status. Since cumulative organ damage and toxicity of therapy may affect these patients, we also included a cohort of At Risk individuals as previously described. ${ }^{1}$

Methods We assessed three cohorts: (1) patients with established AI-CTD (SLE, Sjogrens syndrome or undifferentiated CTD >12 months); (2) at risk individuals referred to secondary care due to ANA +and symptoms suggestive of AI-CTD less than 12 months duration; (3) age and sex matched healthy controls. Cognitive dysfunction was tested using the MoCA. Cardiovascular risk was assessed by recording diabetes, hypertension, previous angina or AMI, atrial fibrillation and cholesterol. Type I interferon activity was assessed using a validated two-score system for IFN status previously described. $^{2}$

Results As expected, the number of patients with an abnormal MOCA score was greater in AI-CTD and At-Risk individuals than in the healthy controls (HC: 20\%; At Risk: 39\%; SLE: $34 \%)$. Also as expected, the IFN scores varied significantly between these groups $(\mathrm{p}=0.046, \mathrm{~F}=4.66)$.

We compared parameters between individuals with normal and abnormal MoCA scores within each group and in all groups. Results are shown in table 1 .

In patients with an AI-CTD, the cognitive function assessed wasnt associated to any of the immune-related but associated

Abstract 280 Table 1 Results

\begin{tabular}{|c|c|c|c|c|c|c|c|c|c|}
\hline \multirow[t]{2}{*}{ Parameter } & \multicolumn{3}{|c|}{ At Risk } & \multicolumn{3}{|c|}{ Al-CTD } & \multicolumn{3}{|c|}{ All patients } \\
\hline & No $C D(n=17)$ & $C D(n=11)$ & $\mathrm{p}$ & No $C D(n=21)$ & $C D(n=11)$ & $\mathrm{p}$ & No $C D(n=50)$ & $C D(n=25)$ & $\mathrm{p}$ \\
\hline Age & $\begin{array}{c}58.47 \text { (SD } \\
12.41)\end{array}$ & 58.09 (SD17.81) & $\mathrm{ns}$ & 44.5 (SD 12.71) & 55 (SD 14.71) & 0.06 & $\begin{array}{c}50,98 \text { (SD } \\
15.17)\end{array}$ & $\begin{array}{c}55.96 \text { (SD } \\
15.13)\end{array}$ & $\mathrm{ns}$ \\
\hline Gender (female) & $94 \%$ & $63 \%$ & 0.04 & $73 \%$ & $70 \%$ & $\mathrm{~ns}$ & $83 \%$ & $70 \%$ & ns \\
\hline 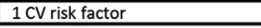 & $35 \%$ & $54 \%$ & $\mathrm{~ns}$ & $19 \%$ & $54 \%$ & 0.04 & $32 \%$ & $52 \%$ & 0.06 \\
\hline Fibromyalgia & $17.6 \%$ & $18 \%$ & $\mathrm{~ns}$ & $14.3 \%$ & $0 \%$ & ns & $20.4 \%$ & $8 \%$ & ns \\
\hline Neuropsychiatric Manifestations & $11.7 \%$ & $9 \%$ & $\mathrm{~ns}$ & $5 \%$ & $0 \%$ & ns & $8 \%$ & $4.5 \%$ & ns \\
\hline Current glucocorticoids & $0 \%$ & $0 \%$ & ns & $23.8 \%$ & $9 \%$ & $\mathrm{~ns}$ & $13 \%$ & $4.5 \%$ & ns \\
\hline Smoking & $12.5 \%$ & $12.5 \%$ & $\mathrm{~ns}$ & $25 \%$ & $9 \%$ & $\mathrm{~ns}$ & $16.2 \%$ & $9 \%$ & $\mathrm{~ns}$ \\
\hline $\lg G$ & 11.3 (SD 2.29) & 13 (SD 2.47) & ns & 13.65 (SD 3.88) & 14.42 (SD 8.94) & ns & \begin{tabular}{|l}
12.47 (SD 3.46) \\
\end{tabular} & 13.55 (SD 5.91) & ns \\
\hline ESR & $8(S D 2.64)$ & - & - & $\begin{array}{c}20.43 \text { (SD } \\
21.47)\end{array}$ & 11 (SD 8.44) & ns & 16.70 & 11 & ns \\
\hline C3 & 1.29 (SD 0.33) & $1.37(\mathrm{SD} 0.18)$ & ns & 1.31 (SD 0.34) & 1.24 (SD 0.49) & $\mathrm{ns}$ & $1.30($ SD 0.33$)$ & 1.32 (SD 0.33) & ns \\
\hline C4 & 0.24 (SD 0.09) & 0.25 (SD 0.06) & ns & 0.25 (SD 0.11) & 0.26 (SD 0.1) & ns & 0.25 (SD 0.1) & 0.25 (SD 0.07 & $\mathrm{~ns}$ \\
\hline Number of ENA & 1.17 (SD 0.72) & 1.27 (SD 0.9) & Ns & 1.66 (SD 1.74) & 1.27 (SD 1.27) & $\mathrm{ns}$ & 1.44 (SD 1.39) & 1.27 (SD 1.07) & ns \\
\hline Physician VAS & 0.38 (SD 0.66) & 1.05 (SD 1) & $\mathrm{ns}$ & 2.25 (SD 2.06) & 1.72 (SD 1.11) & ns & 1.37 (SD 1.81) & 1.37 (SD 1.08) & ns \\
\hline Patient VAS & 3.5 (SD 2.5) & 4.85 (SD 3.02) & $\mathrm{ns}$ & 4.17 (SD 2.5) & 4.6 (SD 3.1) & $\mathrm{ns}$ & $3.83($ SD 2.43) & 4.80 (SD 2.89) & $\mathrm{ns}$ \\
\hline \begin{tabular}{|l|} 
Education $<12 \mathrm{y}$ \\
\end{tabular} & $20 \%$ & $36 \%$ & $\mathrm{~ns}$ & $17.6 \%$ & $50 \%$ & $\mathrm{~ns}$ & $13.6 \%$ & $37.5 \%$ & 0.023 \\
\hline Currently employed & $21.4 \%$ & $20 \%$ & ns & $85 \%$ & $40 \%$ & 0.019 & $53 \%$ & $28.5 \%$ & ns \\
\hline \begin{tabular}{|l|} 
Anxiety or depression \\
\end{tabular} & $33.3 \%$ & $72.7 \%$ & 0.047 & $60 \%$ & $50 \%$ & ns & $57.5 \%$ & $58.3 \%$ & ns \\
\hline IFN Score A & 6.23 (SD 1) & 5.48 (SD 1.26) & $\mathrm{ns}$ & 5.22 (SD 1.22) & 5.15 (SD 1.95) & $\mathrm{ns}$ & 5.64 (SD 1.22) & 5.32 (SD 1.61) & $\mathrm{ns}$ \\
\hline IFN Score B & 6.49 (SD 0.9) & 5.87 (SD 0.89) & ns & 5.7 (SD 1.15) & 6.27 (SD 1.46) & $\mathrm{ns}$ & 6.02 (SD 1.11) & 6.07 (SD 1.2) & ns \\
\hline
\end{tabular}


with the presence of a cardiovascular risk factor $(p=0.04)$ while $\mathrm{CD}$ was associated with anxiety and depression in at risk individuals $(p=0.047)$. A relationship between $C D$ and level of education, gender and current work was also observed.

Conclusions In this exploratory study we identified an association between conventional cardiovascular risk factors and cognitive dysfunction. However there was no association between any of the immune parameters and MoCA score. Prevention of cognitive dysfunction in SLE should focus on early identification and treatment of cardiovascular risk.

Funding Source(s): None

\section{REFERENCES}

1. Md Yusof, et al. ARD 2018.

2. El-Sherbiny, et al. Sci Rep 2018.

\section{LUPUS NEPHRITIS BIOMARKERS}

${ }^{1}$ Safak Mirioglu, ${ }^{2}$ Suzan Çnar, ${ }^{3}$ Halil Yazc, ${ }^{4}$ Ahmet Gül, ${ }^{4}$ Lale Öcal, ${ }^{4}$ Murat Nanç, ${ }^{4}$ Bahar Artm Esen*. 'Istanbul University, Istanbul Faculty of Medicine, Department of Internal Medicine; ${ }^{2}$ Stanbul University, Experimental Research Institute; ${ }^{3}$ stanbul University, Istanbul Faculty of Medicine, Department of Internal Medicine, Division of Nephrology; ${ }^{4}$ Istanbul University, Istanbul Faculty of Medicine, Department of Internal Medicine, Division of Rheumatology

\subsection{6/lupus-2019-|sm.281}

Background TWEAK, MCP-1 and NGAL, mediators in pathogenesis of systemic lupus erythematosus (SLE), are proinflammatory cytokines/chemokines that are thought as potential biomarkers reflecting disease activity. In this study, we aimed to investigate the association of serum (s) and urine (u) levels of TWEAK, MCP-1 and NGAL with disease activity in both renal and non-renal SLE.

Methods Thirty active patients with SLE (15 renal and 15 non-renal) were recruited. Thirty-one inactive patients with SLE (16 renal and 15 non-renal), 14 patients with ANCAassociated vasculitis (AAV) all of whom had active renal involvement and 20 healthy volunteers were selected as control groups. Serum and urine levels of TWEAK, MCP-1 and NGAL were tested using ELISA.

Results Sixty-one SLE patients, 51 (83.6\%) of whom were female, with a median disease duration of 83 (23.5-135) months and a median age of $35(27-47.5)$ were included in the study. Serum and urine levels of TWEAK and NGAL were significantly higher in the active SLE group compared with the inactive SLE $(n=31)$ group (sTWEAK: $\mathrm{p}=0.005$; uTWEAK: $\mathrm{p}=0.026$; sNGAL: $\mathrm{p}<0.001$; uNGAL: $\mathrm{p}=0.002)$; whilst no significant differences regarding serum and urine MCP-1 levels were observed $(p=0.189$ and $p=0.106)$. uTWEAK $\quad(p=0.237), \quad s M C P-1 \quad(p=0.141)$, uMCP-1 $\quad(p=0.206), \quad$ sNGAL $\quad(p=0.419)$ and uNGAL $(p=0.443)$ levels did not differ between patients with active LN and non-renal active SLE; yet levels of sTWEAK were higher in patients with active LN $(p=0.006)$. There were no differences between active $\mathrm{LN}$ and renal active AAV. Levels of all biomarkers were correlated with SLEDAI (sTWEAK: $p=0.001$; uTWEAK: $p=0.006$; sMCP-1: $\mathrm{p}=0.049 ;$ uMCP-1: $\mathrm{p}=0.014 ;$ sNGAL: $\mathrm{p}<0.001$; uNGAL: $\mathrm{p}=0.002)$.
Conclusions sTWEAK, uTWEAK, sNGAL and uNGAL are significant biomarkers showing disease activity in SLE. However, our results implicate that these biomarkers may not be specific for SLE, and can be elevated in patients with active renal involvement of AAV. sTWEAK may be of use for discriminating active nephritis from non-renal active disease in SLE. Further studies are awaited to confirm these Results

Funding Source(s): This study was funded by Istanbul University with the project number TTU-2017-24 738

\section{GENERATION OF HYDROLYZED COMPLEMENT COMPONENT C3 IS SUBSTANTIALLY ELEVATED IN SLE}

Michelle Elvington, M Kathryn Liszewski, John P Atkinson, Alfred H Kim*. Washington University School of Medicine

\subsection{6/lupus-2019-|sm.282}

Background Complement activation is a central pathophysiologic event in several autoimmune diseases. A key activation event is the conversion of native $\mathrm{C} 3$ to $\mathrm{C} 3(\mathrm{H} 2 \mathrm{O})$, where a highly reactive thioester bond in $\mathrm{C} 3$ is hydrolyzed. $\mathrm{C} 3(\mathrm{H} 2 \mathrm{O})$ can be utilized to generate C3 convertase, which further drives complement activation via the alternative pathway. C3 (H2O) has been elusive to measure, but we have recently developed a novel ELISA-based assay allowing for its accurate measurement. We hypothesized that in autoimmune diseases where complement activation is a central feature, $\mathrm{C} 3(\mathrm{H} 2 \mathrm{O})$ levels will be elevated reflecting a primed state for triggering inappropriate alternative pathway activation and amplification. Methods Healthy adult subjects $(\mathrm{n}=5)$ and adults with classified SLE $(n=6)$ were enrolled and consented for serum collection at Washington University School of Medicine. Frozen serum was tested either immediately after thawing or after incubation at room temperature or $37^{\circ} \mathrm{C}$ for 6 or 24 hours. $\mathrm{C} 3(\mathrm{H} 2 \mathrm{O})$ was measured by an ELISA assay that utilizes a capture antibody to $\mathrm{C} 3 \mathrm{~b}$ and a detection antibody to C3a. Data was analyzed using Prism version $7.0 \mathrm{~d}$ (GraphPad Software, Inc.). Statistical significance was determined using 2-way ANOVA with Dunns multiple comparisons test.

Results Substantial elevation of serum C3(H2O) levels were observed from patients with SLE following incubation at RT and $37^{\circ} \mathrm{C}$ compared to healthy controls. The differences were most pronounced in samples incubated at $37^{\circ} \mathrm{C}$ for 6 hours, with over a 4-fold increase in $\mathrm{C} 3(\mathrm{H} 2 \mathrm{O})$ levels in both the SLE samples. Interestingly, sera from both groups (healthy controls and SLE) did not reflect any difference in baseline $\mathrm{C} 3(\mathrm{H} 2 \mathrm{O})$ levels.

Conclusions Both SLE and RA are associated with elevated C3 (H2O) levels following in vitro incubation compared to healthy controls. These data suggest that the potential for C3 (H2O) formation in SLE and RA patients are higher compared to healthy controls, which could support additional complement activation or utilization of $\mathrm{C} 3(\mathrm{H} 2 \mathrm{O})$ in other pathways such as intracellular activation in immune cells. Thus, In addition to a possible diagnostic tool for pathogenic autoimmunity, these data may also suggest novel mechanisms of how complement could drive symptomatic autoimmune disease.

Funding Source(s): NIH R01 GM0099111 